\title{
Wear of micro diamond tool in ultra-precision turning under dry and minimum quantity lubrication conditions
}

W J Zong ( $\square$ zongwenjun@163.com )

Harbin Institute of Technology https://orcid.org/0000-0002-3960-7323

Guilin Zhuang

Harbin Institute of Technology

Yifu Tang

Harbin Institute of Technology

\section{Research Article}

Keywords: minimum quantity lubrication, micro diamond tool, cutting temperature, cutting fluid

Posted Date: February 11th, 2022

DOI: https://doi.org/10.21203/rs.3.rs-1336909/v1

License: (c) (i) This work is licensed under a Creative Commons Attribution 4.0 International License.

Read Full License 


\title{
Wear of micro diamond tool in ultra-precision turning under dry and minimum quantity lubrication conditions \\ Guilin Zhuang, Wenjun Zong ${ }^{*}$,Yifu Tang \\ Center for Precision Engineering, Harbin Institute of Technology, Harbin, 150001, P.R. China
}

\begin{abstract}
Cutting force and cutting heat are two main reasons for the wear of diamond tools. An effective way to reduce the cutting temperature is minimum quantity lubrication (MQL). To explore the effect of MQL on the cutting temperature, a calculation model for the cutting temperature of micro diamond tool under dry friction condition is established firstly by using the Fourier's law of heat conduction, through which the maximum temperature of the tool can be acquired. Regarding the boundary film as a layer of heat-conducting medium, a revised calculation model for the temperature distribution on tool rake face under MQL condition with different cutting fluids is further established. As expected, the wear volume of micro diamond tool can be successfully predicted by using the Usui wear rate model in response to different cutting fluids and different cutting distances. Finally, cutting experiments with different cutting fluids are performed to verify the established models. The experimental observations agree well with the theoretical prediction results. Such satisfactory consistency confirms that the cutting fluid with low viscosity can reduce the cutting temperature and inhibit tool wear effectively.
\end{abstract}

Keywords: minimum quantity lubrication; micro diamond tool; cutting temperature; cutting fluid

\section{Introduction}

Micro-cutting technology is usually used for manufacturing small-size parts, in which micro diamond tools are indispensable. In this work, micro diamond tools refer to the diamond tools with a nose radius of less than $100 \mu \mathrm{m}$. Due to the small nose radius, the preparation of micro diamond tools is much more difficult than the conventional diamond tools, which requires higher precision machine tools for sharpening. Especially for the tool with micro nose radius of less than $30 \mu \mathrm{m}$, the sharpening process is required to be quite stable. This is because small changes of sharpening parameters may produce large profile errors on the cutting edge [1]. With the development of micro-nano manufacturing technology, high-efficiency and low-cost processing has become the urgent requirement in different industrial sectors. Therefore, more rigorous wear resistance is put forward for the micro diamond tools. As well known in micro-

\footnotetext{
* Corresponding author. Tel: +86-0451-86412924 Fax: +86-0451-86415244

E-mail: zongwenjun@163.com (Wenjun Zong)
} 
cutting, the depth of cut and feed rate are extremely small, which will introduce the size effect and increase the cutting ratio significantly. Meanwhile, a large amount of heat flux will be generated, which eventually increases the temperature of the tool itself due to its small heat capacity. An elevated cutting temperature inevitably accelerates tool wear. Therefore, in microcutting, the excellent wear resistance, thermal hardness, impact resistance and other aspects of the tool are necessary.

Micro-cutting is a complex plastic deformation process, and deformation generates cutting force and cutting heat. In the cutting process, the plastic deformation of chips, tool-chip friction, and elastic recovery of workpiece surface will all cause the increase of cutting temperature. When the cutting temperature rises to the internal bond energy of diamond, it will lead to the fracture of carbon-carbon bond, causing the wear of cutting edge, which affects the life of micro diamond tool. In addition, the wear of tool further increases the cutting force, elastic recovery, chip deformation and cutting temperature, which in turn promotes the increment of tool wear. Therefore, the amount of cutting heat flux and cutting heat distribution, especially the conduction of cutting heat in the tool, are always the hot issues in micro-cutting. In fact, cutting heat has a great influence on the tool wear rate, surface accuracy of workpiece, as well as the surface integrity of microstructure. Therefore, the research on cutting heat is of great significance for micro-cutting technology.

Up to now, there are three methods to model the cutting heat, i.e. analytical modeling [2], digital modeling [3], and hybrid modeling [4]. Analytical modeling is to find the relationship between various variables and establish the corresponding mathematical model. Digital modeling depends on the finite element simulation. With the increasing accuracy of material constitutive models, the digital modeling method has been applied more and more, which can direct the actual machining. Hybrid modeling is a combination method of analytical and digital modeling.

Komanduri and Hou provided a comprehensive review of recent advances in cutting 
temperature measurement methods, and claimed that the accurate prediction of cutting temperature field and thermal distribution remains a major challenge. This is because that the cutting temperature measurement during machining is very difficult, which is a challenge for any model validation [5]. Abukhshim et al. concluded that as the cutting velocity increases, the heat transferred to the tool increases due to the increased contact area between the tool and the chip. In terms of their experimental observations, the percentage of cutting heat transferred to the tool will reach $65 \%$ at a cutting velocity of $1200 \mathrm{~m} / \mathrm{min}$ [6]. In order to predict the cutting temperature distribution on tool rake and flank faces, Grzesik used a special algorithm of the finite difference method and the method of elementary balance (MEB) to analyze the temperature in machining of C45 carbon steel with uncoated and coated ISO P20 carbide tools at a cutting velocity of 72-145 m/min, which yielded a good prediction accuracy (6\%) [7].

Wang et al. determined the heat distribution ratio according to the lengths of adhesion zone and sliding zone, and finally established a temperature prediction model with consideration of non-uniform heat intensity distribution at the tool-chip contact interface [8]. Sawangsri et al. concluded that in micro-cutting of aluminum alloy, the ratio of cutting heat distributed in the tool, tip area and workpiece is $8: 8.5: 1$. In cutting of single crystal silicon, however, the cutting heat distribution ratio is $14: 16: 1$. For the titanium alloy, the ratio of cutting heat distributed in the tool, tip, chip and workpiece becomes 350:460:1.4:1 [9]. Davies et al. compared the measured temperature with the finite element method predicted temperature and found large difference in the results acquired by these two methods. They concluded that such difference is caused by two reasons, i.e. the error of modeling method and the error of non-deterministic factors in experiment [10]. Zhao [11] explored the effect of AlTiN coating on the cutting heat distribution coefficient and cutting temperature increment in orthogonal cutting of Inconel 718.

Finite element method (FEM) is effective in predicting the distribution of cutting temperature. Puls et al. developed a FEM-based simulation model to analyze the heat distribution in dry metal cutting [12]. You et al. employed the Taguchi method to determine the factors affecting 
the turning process. They found that in machining of titanium alloys, the reason of tool wear is chip adhesion dependent on the high cutting temperature [13]. Hao et al. carried out a lot of friction tests at high temperature to emulate the hard machining of $\mathrm{H} 13$ hardened steel by using TiAlN coated tool and uncoated tool, respectively [14]. Su et al. discussed on the variations of cutting temperature, tool-to-cutting chip contact temperature and tool-to-workpiece contact temperature with tool temperature in light of tool wear and surface integrity [15]. Meng et al. developed a 2D FEM orthogonal cutting model for the analysis of high-speed milling of ADC12 aluminium alloy [16]. Karaguzel et al. presented a new approach for modelling and measuring the change of tool temperature in milling. This new model can predict the effect of milling conditions on the cutting temperature and, in particular, determine the relationship between the tool temperature and the depth of cut [17]. The above articles all concentrated on the modelling of cutting temperature field under dry cutting condition. There is no research to compare the temperature field under different cutting fluids and reveal the inhibiting effect of different cutting fluids on the wear of diamond tool.

This work aims to develop the suppression method for the wear of micro diamond tools from the perspective of reducing cutting heat and improving tool strength. Firstly, the wear mechanism of micro diamond tools in cutting of 6061 aluminum alloy is explored. Secondly, the models to predict the temperature distribution of tool under dry friction and MQL conditions are established respectively. As expected, the highest temperatures of tool under dry friction and different cutting fluids are calculated, which is used to select the best cutting fluid for the suppression of tool wear.

\section{Theoretical model of cutting temperature}

\subsection{Generation of cutting heat}

In micro-cutting, the wear of diamond tool is usually resulted from the cutting heat and cutting force, which are complementary with each other. For instance, when diamond tool with 
a large nose radius more than $100 \mu \mathrm{m}$ cuts aluminum alloy, the cutting edge mainly suffer from the micro-crack wear and thermochemical wear caused by the cutting heat and cutting force. Unfortunately, there is a lack of research on the mechanism of cutting heat generation when using micro diamond tool. Previous studies revealed that during micro-cutting, different workpiece materials, different cutting velocities and different tool parameters will change the amount of cutting heat as generated.

The workpiece materials undergo shear deformation and elastic-plastic deformation under the cutting action of micro diamond tool, resulting in the deformation resistance force, i.e. cutting force. The cutting force produces deformation work, and the deformation work generates cutting heat. In addition, the friction between the tool rake face and the chip surface, and the friction between the tool flank face and the machined surface will also generate heat. Therefore, there are three heat sources in response to the three deformation zones in the cutting process, as shown in Fig. 1. The first source is the shear deformation appearing in the primary deformation zone. The second source is the friction heat due to the contact between the rake face and the chip in the secondary deformation zone. The third source is also the friction heat due to the contact between the flank face and the machined surface in the tertiary deformation zone.

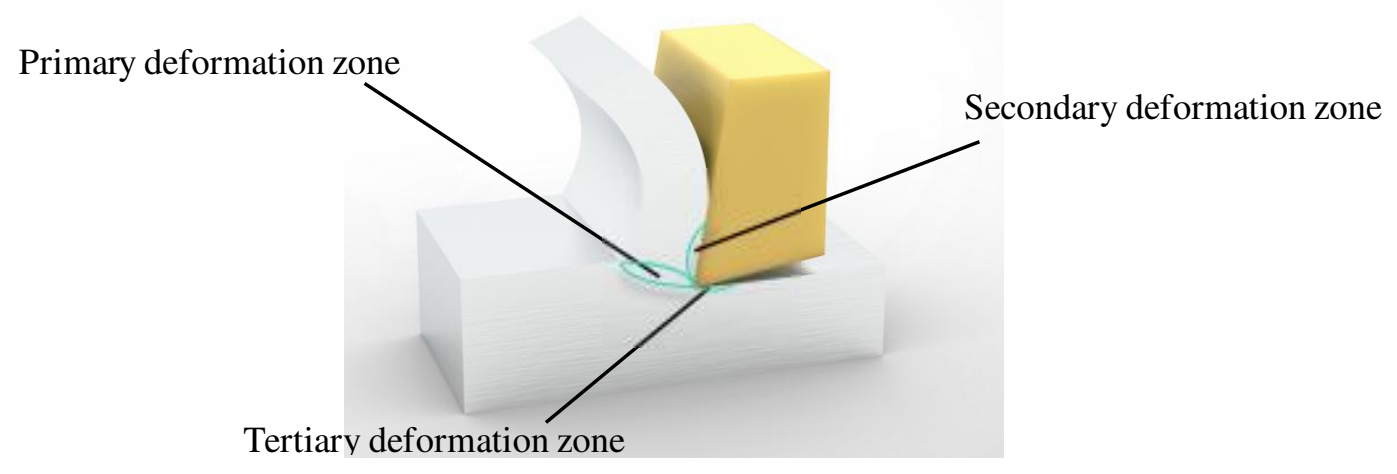

Fig. 1 Deformation zones and cutting heat resources in micro-cutting.

\subsection{Modeling on the cutting heat}

As illustrated in Fig.1, three deformation zones jointly generate the cutting heat. In dry 
cutting, the cutting heat mainly distributes in the chip, workpiece and tool. Here, the heat fluxes in the chip, workpiece and tool are denoted as $Q_{C}, Q_{W}$ and $Q_{T}$, respectively. Since the heat flux generated in the third deformation zone is tiny and can be ignored, the total heat flux $E_{Q}$ is formulated as

$$
E_{Q}=Q_{C}+Q_{W}
$$

For the heat flux in the chip, heat generated in the first deformation zone $Q_{C}$ is calculated as

$$
Q_{C}=F_{c} \cdot v_{c}
$$

where $v_{c}$ is the shear velocity in the first deformation zone, and $F_{c}$ is the shear force in the first deformation zone.

$F_{c}$ is written as

$$
F_{c}=d_{u} \int_{0}^{l_{s}} \tau_{s} d x
$$

where $\tau_{s}$ is the shear stress, $A_{s}$ is the shear area.

Likewise, $Q_{W}$ is given by

$$
Q_{W}=F_{f} \cdot v_{s h}
$$

where $v_{s h}$ is the relative velocity between the chip and tool. $F_{f}$ is the friction force in the second deformation zone.

$F_{f}$ is expressed as

$$
F_{f}=\left(1-2^{-a}+\frac{2^{a+1}-1}{2^{a}(a+1)}\right) \cdot \mu \cdot \sigma_{s 2} \cdot \frac{\cos \left(\alpha_{r}-\phi\right) \cdot t_{u} \cdot d_{u}}{\sin \phi}
$$

where $a$ is the power index. $\mu$ is the coefficient of friction. $\alpha_{r}$ is the effective rake angle of micro diamond tool. $\phi$ is the shear angle. $t_{u}$ is the depth of cut. $d_{u}$ is the cutting width. The heat generated in the first and second deformation zones will change the temperature 
distribution. The temperature rise in the first deformation zone caused by the shear deformation is empirically calculated as [18]

$$
\theta_{s}=\frac{\tau_{s}}{c_{w} \cdot \rho_{w} \cdot \tan \phi} \operatorname{erf} \sqrt{\frac{R \cdot \tan \phi}{4}}
$$

where erf is the error function.

In Eq. (3), the expression of $R$ is

$$
R=\frac{c_{w} \cdot \rho_{w} \cdot v_{c} \cdot h}{k_{w}}
$$

where $k_{w}$ is the thermal conductivity of workpiece. $c_{w}$ is the heat capacity per unit volume. $\rho_{w}$ is the density of workpiece. $h$ is the undeformed chip thickness. $v_{c}$ is the cutting velocity.

The temperature distributed in the second deformation zone is affected by the plastic deformation in the first deformation zone and the friction effect between the tool and the chip. The heat from the second deformation zone is transferred into the micro diamond tool from the rake face, and the conduction pattern of which is in accordance with the Fourier's law. In this work, the Fourier heat transfer law is the theoretical basis to determine the temperature field in the second deformation zone or inside the tool.

\subsection{Modeling on the cutting temperature field under different conditions}

Minimum quantity lubrication (MQL) technology is a promising cooling and lubrication method in metal cutting. In this work, such cooling and lubrication method was also used to suppress tool wear. Under the condition of MQL, the contact state between the tool and the chip can be divided into two types, i.e. boundary film contact and no boundary film contact [19]. Under the influence of boundary film contact state, the friction coefficient at the tool-chip interface changes, resulting in a reduction in friction, which in turn leads to the variation of temperature field in the cutting area or inside the tool. Therefore, the establishment of cutting temperature field under the boundary film condition is very important for exploring the best method to suppress tool wear. Up to now, little models of the cutting temperature field under 
MQL have been reported. Therefore, in this work the cutting temperature field models under dry cutting and boundary film conditions were established according to the Fourier's law of heat conduction.

\subsubsection{Dry cutting condition}

For the dry cutting mode, no lubrication is used. In this condition, the chip and tool rake face contact directly, and the friction heat is transferred into the tool from the rake face. Moreover, the cutting heat generated in the first deformation zone will also be transferred into the tool by conduction. According to the Fourier law of heat conduction and based on the relationship between tool cutting temperature and wear volume to be established in this paper, three assumptions should be made before establishing the cutting temperature model. Firstly, the generation and distribution of heat sources are in a steady-state. Secondly, all the energy generated in the second deformation zone is converted into heat, ignoring the energy used for phase change within the material. Thirdly, as the maximum temperature is considered in this paper, in order to simplify the model, the heat conduction in the second deformation zone is one-dimensional heat conduction.

The heat flux per unit area of the second deformation zone is expressed as

$$
q_{0}=F_{f} \cdot V_{s h} / A_{s h}
$$

where $F_{f}$ is the friction force of tool-chip in dry cutting. $V_{s h}$ is the sliding velocity of chip relative to the rake face. $A_{s h}$ is the tool-chip contact area.

The nose radius of micro diamond tools is usually no more than $100 \mu \mathrm{m}$, which far less than the diamond thickness of tool. Therefore, a one-dimensional heat transfer model is proposed to represent the heat transfer state of the tool. In light of the Fourier law of heat conduction, when the micro diamond tool is in the state of dry friction, the one-dimensional heat transfer function can be formulated as 


$$
\frac{\partial \theta}{\partial t}-\kappa \frac{\partial^{2} \theta}{\partial x^{2}}=0
$$

where $\kappa$ is the thermal diffusivity of tool material (diamond).

As Eq. (6) involves time variables, it can be solved using the Laplace transform.

Supposed that the diamond thickness of tool is $l$ and the original temperature of tool is $20^{\circ} \mathrm{C}$, the boundary condition of the partial differential equation is

$$
\left\{\begin{array}{l}
\left.\frac{\partial \theta}{\partial x}\right|_{x=0}=-\frac{q_{0}}{k} \\
\theta(l, t)=20
\end{array}\right.
$$

where $k$ is the tool's thermal conductivity.

To improve the accuracy of the model, the Laplace transformation is employed to obtain the analytical solution of Eq. (7). Regarding $x$ as a parameter and performing the Laplace transformation for the independent variables $t$ on both sides of Eq. (7) can yield

$$
L(\theta(x, t))=\hat{\theta}(x, p)
$$

where $L$ is the Laplace transform form, $x$ is the distance, assuming $p$ as a constant.

The Laplace transformation is also performed on the boundary conditions, which produces the following ordinary differential equation

$$
\hat{\theta_{x x}}=\frac{1}{\kappa} \hat{\theta}(x, p)
$$

The definite solution problem of Eq. (7) can be solved. The general solution is given by

$$
\hat{\theta}(x, p)=c_{1}(p) e^{\sqrt{p \cdot \kappa} \cdot x}+c_{2}(p) e^{-\sqrt{p \cdot \kappa} \cdot x}
$$

Eq. (10) can be simplified as

$$
\hat{\theta}(x, p)=\hat{g}(p) \cdot e^{-\sqrt{p \cdot \kappa} \cdot x}
$$

Regarding $x$ as a parameter and calculating convolution on both sides of Eq. (11) can output the Laplace inverse transformation, i.e. 


$$
L^{-1}\left[e^{-\sqrt{\kappa \cdot p} \cdot x}\right]=\frac{x \cdot \sqrt{\kappa}}{2 \sqrt{\pi} \cdot t^{3 / 2}} \cdot e^{-\frac{x^{2} \kappa}{4 \cdot t}}
$$

Therefore, the temperature distribution on the rake face due to the friction heat from the second deformation zone is calculated as

$$
\theta_{m}(x, t)=\frac{q_{0}}{k} \cdot \sum_{i=1}^{n}(-1)^{n} \cdot \operatorname{erfc} \frac{2 n l+x}{2 \sqrt{\kappa \cdot t}}+\frac{q_{0}}{k} \cdot \sum_{i=1}^{n}(-1)^{n} \cdot \operatorname{erfc} \frac{2 n l-x}{2 \sqrt{\kappa \cdot t}}
$$

where $l$ is thr length of contact between the rake face of the tool and the chip.

The temperature distributed on the rake face is related to not only the friction heat in the second deformation zone, but also the shear deformation in the first deformation zone. Therefore, the maximum temperature on the rake face can be modified as

$$
\theta_{\max }=\theta_{m \max }+\theta_{s 0}+\theta_{0}
$$

where $\theta_{\text {mmax }}$ is the maximal temperature dependent on the friction heat in the second defmation zone. $\theta_{s 0}$ is the temperature rise in the second deformation zone caused by the plastic deformation in the first deformation zone. $\theta_{0}$ is the ambient temperature.

\subsubsection{Boundary film lubrication}

MQL is a green lubrication method and has some unmated advantages, such as low energy consumption, low cost, and less environmental pollution [20]. It has become the preferred cooling method in the cutting operation. The specific structure of the MQL unit is shown in Fig. 2. The MQL unit supplies the cutting fluid to the cutting area as water droplets with a tiny radius. The micro-defects on the surfaces of tool and chip form capillary tubes, through which the cutting fluid is brought to the tool-chip contact area by capillary action. The lubricant forms a boundary film between the tool and chip, which serves to reduce the coefficient of friction and can be thought of as a layer of medium for heat transfer. Due to the limited amount of lubricant sprayed, the oil film does not fill the entire tool-chip contact surface. Therefore, under MQL condition, the cutting fluid does not act on the entire tool-chip contact area, which will be divided into two parts, i.e. dry friction and boundary lubrication regions. We will model the 
heat transfer for the boundary film and dry friction cases separately, and then weight the average according to the proportion of both, followed by the lubricant with the better temperature reduction effect. First we have to obtain the proportion of the boundary film situation on the tool-chip contact surface. According to previous work [21], the ratio of boundary lubrication area to total contact area is given by

$$
\beta=e^{\left(-\frac{t_{x}}{t_{r}}\right)}
$$

where $t_{x}$ is the cutting velocity $v$ dependent time over a distance of $x$, and $t_{x}=x / v . t_{r}$ is the average time that the adsorbed molecules stay on the surface.

$t_{r}$ is expressed as

$$
t_{r}=t_{0} \cdot e^{\left(\varepsilon / R \cdot T_{S}\right)}
$$

where $t_{0}$ is the thermal vibration period of the adsorbed molecules, and the direction of which is perpendicular to the processing surface. $\varepsilon$ is the heat of adsorption. $R$ is a constant, and $R=8.314 \mathrm{~J} / \mathrm{mol} \cdot \mathrm{K} . \quad T_{S}$ is the temperature on tool surface during dry friction.

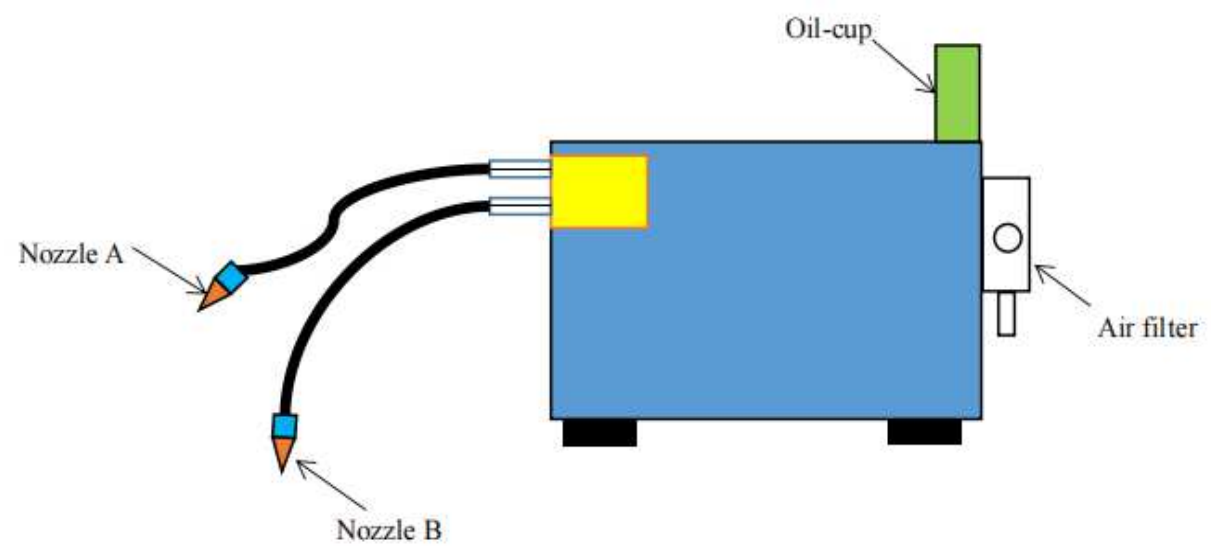

Fig. 2 Schematic diagram of the working principle of the MQL device.

As discussed above, the actual tool-chip contact surface can be divided into dry friction and boundary lubrication regions under MQL condition. Due to the different conduction media, the cutting heat transfer in these two regions is different. Therefore, only the temperature in the 
second deformation zone under lubrication condition with boundary film will be discussed in this work.

In the state of boundary lubrication, an oil film will be formed between the rake face and the chip. Due to the effect of lubricating oil, the friction coefficient between the rake face and the chip is reduced, which reliefs the tool-chip friction and suppresses the generation of cutting heat. In addition, heat is transferred into the tool by both heat conduction and heat convection due to the temperature gradient in the cutting fluid. However, in the field of MQL, conventional lubricants (excluding nanofluids) remove very little heat through thermal convection [22]. In order to simplify the modelling process, the heat flow carried away by the thermal convection is negligible in this work. Therefore, the oil film will be treated as a layer of heat conduction medium. Moreover, it should be further assumed that the heat flux only propagates along with the thickness of the boundary film, and the thickness of the boundary film is equal everywhere. Finally, the Fourier heat conduction equation under the boundary film state is constructed as

$$
\begin{gathered}
\kappa_{c} \cdot \frac{\partial^{2} \theta_{1}}{\partial x^{2}}=\frac{\partial \theta_{1}}{\partial t} \\
\kappa_{s} \cdot \frac{\partial^{2} \theta_{2}}{\partial x^{2}}=\frac{\partial \theta_{2}}{\partial t}
\end{gathered}
$$

The boundary conditions for the contact between the oil film and the chip are given by

$$
\begin{gathered}
k_{c} \frac{\partial \theta_{1}}{\partial x}=-q_{1} \quad x=0, \quad t \geq 0 \\
\theta_{2}(x, t)=0 \quad x \rightarrow \infty, \quad t \geq 0
\end{gathered}
$$

Similarly, the boundary conditions for the oil film and tool contact are written as

$$
\begin{aligned}
\theta_{1}\left(x_{1}, t\right) & =\theta_{2}\left(x_{1}, t\right) \\
-\left.k_{c} \frac{\partial \theta_{1}}{\partial x}\right|_{x=x_{1}} & =-\left.k_{s} \frac{\partial \theta_{2}}{\partial x}\right|_{x=x_{1}}
\end{aligned}
$$

Finally, the Laplace transformation is applied for the time variable $t$ and the Fourier transformation is applied for the position variable $x$, through which the above partial differential 
equations can successfully solve the temperature on tool rake face under the boundary lubrication conditions, i.e.

$$
\begin{gathered}
\theta=q_{1} \cdot \frac{4 \sqrt{k_{c}}}{\kappa_{c} \cdot k_{c}} \cdot \sum_{n=0}^{\infty}(|d|)^{n+1} \cdot \sqrt{t \cdot \pi} \cdot\left\{\exp \left[-\frac{(n+1)^{2} \cdot x_{1}^{2} \cdot \kappa_{c}}{t}\right]-\operatorname{erfc}\left[-\frac{(n+1) x_{1} \cdot \sqrt{\kappa_{c}}}{\sqrt{t}}\right]\right\}+\frac{2 q_{1}}{k_{c}} \sqrt{\frac{t}{\pi \cdot \kappa_{c}}} \\
d=\frac{k_{c} \cdot \kappa_{c} \sqrt{\kappa_{s}}-k_{s} \cdot \kappa_{s} \sqrt{\kappa_{c}}}{k_{c} \cdot \kappa_{c} \sqrt{\kappa_{s}}+k_{s} \cdot \kappa_{s} \sqrt{\kappa_{c}}}
\end{gathered}
$$

where $k_{s}$ is the thermal diffusivity of tool material. $k_{c}$ is the thermal diffusivity of lubrication oil. $t$ is the time. $q_{1}$ is the heat flux per unit area generated by the friction force on the tool-chip interface under the boundary lubrication conditions.

$q_{1}$ is expressed as

$$
q_{1}=F_{f 1} \cdot V_{s h} / A_{s h}
$$

where $F_{f l}$ varies with different lubricants.

The calculated results of temperature rise on tool rake face under different contact conditions are presented in Fig.3. It can be found that under the same cutting parameters, the highest temperature on tool rake face under the boundary lubrication condition is significantly smaller than that achieved under the dry friction condition, which shows that the MQL technology has a good effect on reducing the cutting heat.

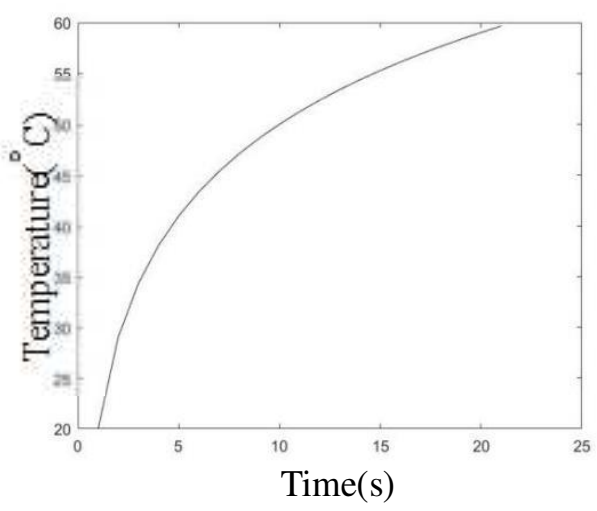

a) dry friction condition

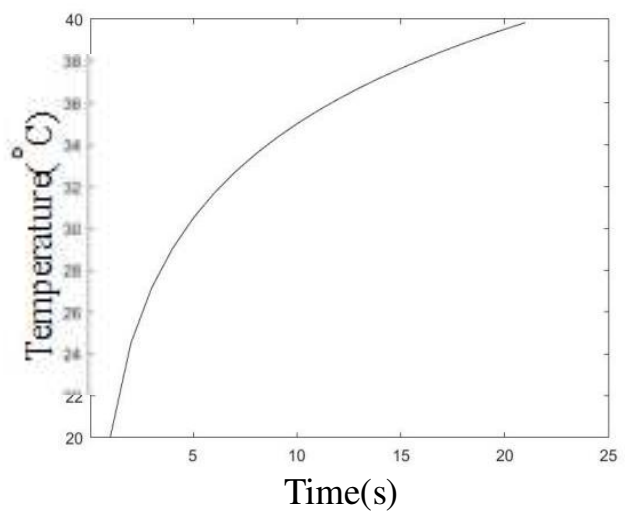

b) boundary lubrication condition

Fig. 3 Tool temperature rise as calculated under different contact conditions.

To simplify the temperature model, it is assumed that the average temperature of tool-chip 
contact region in response to the boundary lubrication condition is $\theta$, and the average temperature of tool-chip contact region in response to the dry friction condition is $\theta_{m}$. In terms of the reported finding [23], the boundary film discretely distributes on the rake face, so the Maxwell-Oaken model is used to calculate the temperature superimposition of these two contact regions. With consideration of the coupling effect of dry friction and boundary film, the average temperature $\bar{\theta}$ of the rake face can be expressed as

$$
\bar{\theta}=\frac{(1-\beta) \cdot(2-\beta) \cdot \theta_{m}+\beta \cdot(3-3 \cdot \beta) \cdot \theta}{2-2 \cdot \beta^{2}}
$$

Because of $0<\beta<1$, Eq. (27) reveals that the temperature of tool rake face under the boundary lubrication condition is significantly lower than the temperature under the dry friction condition, namely $\theta<\theta_{m}$. Therefore, the relationship is fulfilled as follows

$$
\beta \cdot(3-3 \cdot \beta) \cdot \theta<\beta \cdot(3-3 \cdot \beta) \cdot \theta_{m}
$$

Eq. (28) confirms that the temperature of micro diamond tool can be effectively reduced by using the MQL technology.

Due to the small contact area between the flank face and the machined surface, the heat as generated in the third deformation zone is extremely small compared to the other two deformation zones, which can be ignored. Therefore, the cutting heat generated in the third deformation zone was not analyzed in this work.

\subsubsection{The highest temperature on tool rake face under different cutting fluids}

In order to optimize the type of cutting fluid and inhibit tool wear effectively, the temperature variations under different cutting fluids were calculated by using the above established model of cutting temperature field. The cutting fluids include kerosene, absolute ethanol, castor oil, and polyethylene glycol. According to related literature [24], the thermal conductivities of these four cutting fluids are $0.13 \mathrm{~W} / \mathrm{m} \cdot \mathrm{K}, 0.1531 \mathrm{~W} / \mathrm{m} \cdot \mathrm{K}, 0.200 \mathrm{~W} / \mathrm{m} \cdot \mathrm{K}$ and $0.285 \mathrm{~W} / \mathrm{m} \cdot \mathrm{K}$, respectively. Different lubricants lead to a change in the coefficient of friction between the toolchip, which, according to the literature, is obtained as follows: $0.015,0.017,0.0235,0.0278$. 
Under the same cutting parameters, the calculated maximum temperatures on tool rake face are presented in Fig. 4.

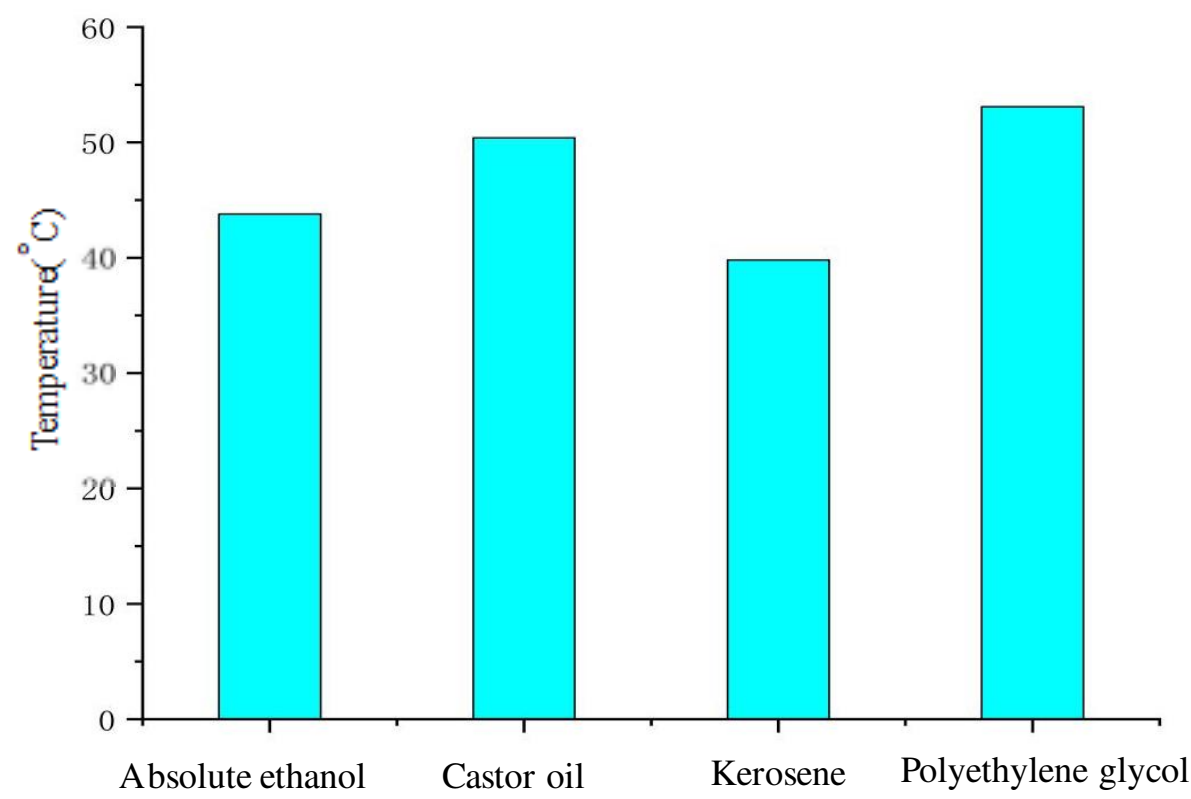

Fig. 4 The maximal temperatures on tool rake face predicted under different cutting fluids and a cutting time of $40 \mathrm{~s}$.

As can be seen from Fig. 4, kerosene has the best cooling effect. This is because kerosene has a low viscosity and a small contact angle on the workpiece material, which is beneficial to the wetting of kerosene on the tool-chip contact face. As a result, the friction between the tool rake face and the chip is relieved. The reductions of friction finally decrease the cutting temperature of tool rake face. For the polyethylene glycol, however, the temperature of rake face is the highest. The reason is that the viscosity of polyethylene glycol is the largest, and the contact angle on the workpiece material is the greatest. It is difficult to spread on the tool-chip contact face. Due to the degradation in reducing the friction and promotion of heat exchange at the tool-chip interface, the highest temperature is resultantly observed on tool rake face when employing the polyethylene glycol as cutting fluid.

According to the Usui wear rate model [25], i.e. Eq. (29), the wear rate of cutting tool is proportional to the exponential power of temperature. The higher the cutting temperature is, the intenser the tool wear does, which means that reducing the temperature of tool is an effective way to suppress the wear of micro diamond tool. 


$$
\frac{\partial W}{\partial t}=M \cdot v_{s h} \cdot \sigma_{n} \cdot \exp \left(-\frac{N}{T}\right)
$$

where $\frac{\partial W}{\partial t}$ is tool wear rate, i.e. the wear volume of tool per unit time. $\sigma_{n}$ is the contact stress in the wear zone. $T$ is the cutting temperature. $M$ and $N$ are the material property dependent constants, and $M=6.524 \times 10^{-11} \mathrm{~m}^{2} \cdot \mathrm{MPa}^{-1}$ and $N=1483 \mathrm{~K} . v_{\text {sh }}$ is the relative sliding velocity between the tool and the chip.

Therefore, when choosing cutting fluids to inhibit the wear of micro diamond tool, the cutting fluids with low viscosity is preferred. Low viscosity lubricant fluids have high wettability (small contact angle), higher mist particle concentration and larger mist particle diameter. Despite their relatively low thermal conductivity, the larger mist particle diameter and higher mist particle concentration reduce surface roughness and inhibit tool wear.

By using Eq. (29), the wear volume of micro diamond tool under different cutting fluids can be successfully calculated, and the results of which are presented in Fig. 5. It can be seen that the wear degree of micro diamond tool is different under different cutting fluids. Anhydrous ethanol and kerosene have the best inhibition to the wear of tool. As discussed above, the anhydrous ethanol and kerosene has a small viscosity, which are beneficial to the reduction of friction at the tool-chip interface. Resultantly, the temperature on the rake face is the lowest, and the tool wear is the lowest too. 


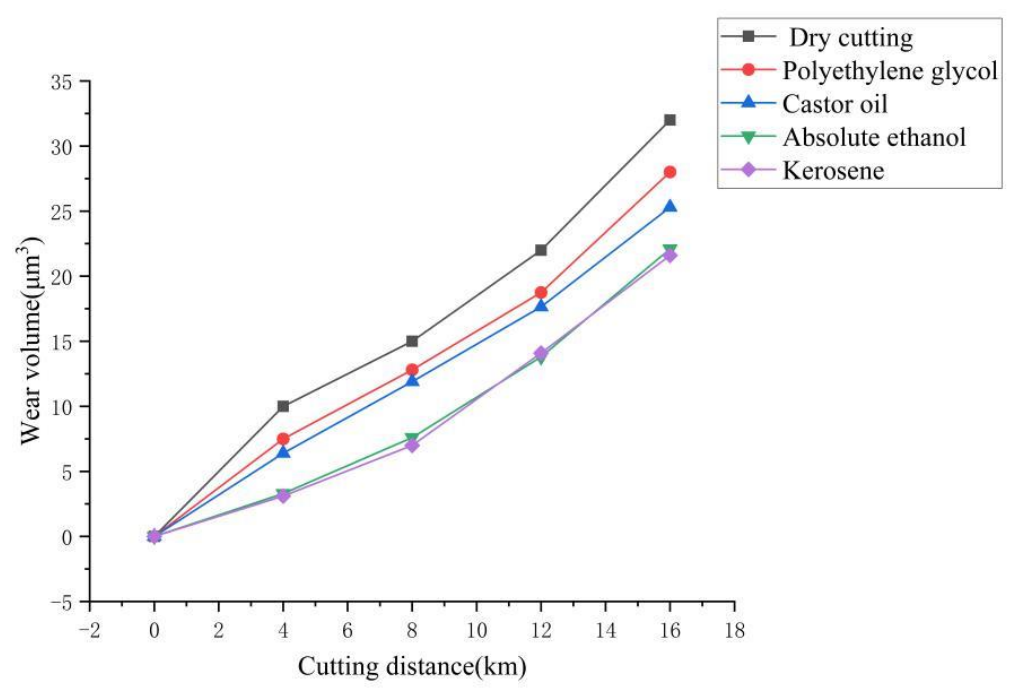

Fig. 5 The wear volume of micro diamond tool predicted under different cutting fluids.

\section{Experimental validations}

\subsection{Preparation of micro diamond tool}

In order to validate the theoretical models as developed above, a micro diamond cutting tool was prepared in a commercial available machine tool, PG3B. The rake face and flank face were both oriented with the (100) crystal plane, and a cylindrical shape was configured for the flank face. Moreover, the specific parameters of the test tool are shown in Table 1.

Table 1 Geometrical parameters of the test micro diamond tool.

\begin{tabular}{cc}
\hline Parameter & Configuration \\
\hline Tool corner nose radius $(\mathrm{mm})$ & 0.05 \\
Opening angle of cutting edge $\left(^{\circ}\right)$ & 100 \\
Rake angle $\left({ }^{\circ}\right)$ & 0 \\
Flank angle $\left({ }^{\circ}\right)$ & 10 \\
\hline
\end{tabular}

The cutting edge waviness and corner nose radius of the prepared micro diamond tool were evaluated by a professional instrument, i.e. the DTRC microscope system. The measuring results are shown in Fig. 6. In Fig. 6, the ideal cutting edge corresponds to the measured corner 
nose radius. The curve fluctuated around the ideal cutting edge is the actual profile of the cutting edge, through which the cutting edge waviness can be evaluated. The results revealed that the test micro diamond tool has a cutting edge waviness of $0.03 \mu \mathrm{m}$ over an opening angle of $80^{\circ}$.

a)

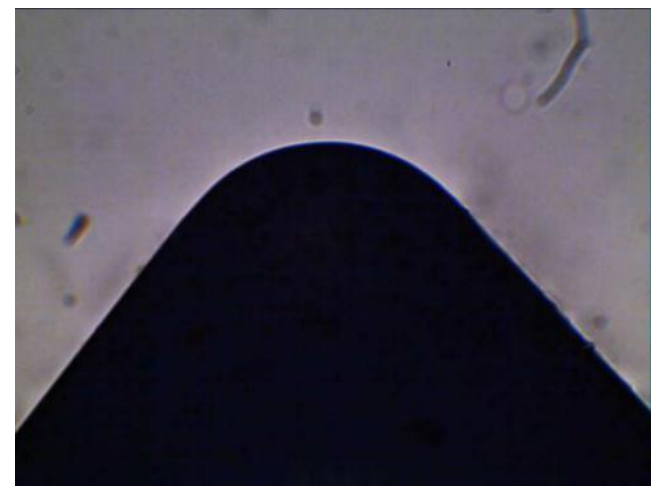

b)

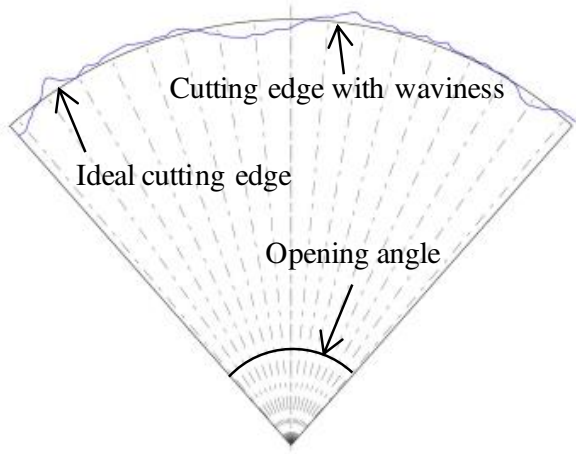

Fig. 6 Evaluation for the prepared micro diamond tool: a) optical image of the cutting edge; b) measured profile of the cutting edge.

The cutting edge radius of the prepared micro diamond tool was evaluated by an atomic force microscope (AFM), i.e. the Nanite B supplied by Nanosurf Ltd. Fig. 7 shows AFM scanning images of 3D topography and 2D cross-section of tool cutting edge, through which a cutting edge radius of $30.9 \mathrm{~nm}$ is read.

a)

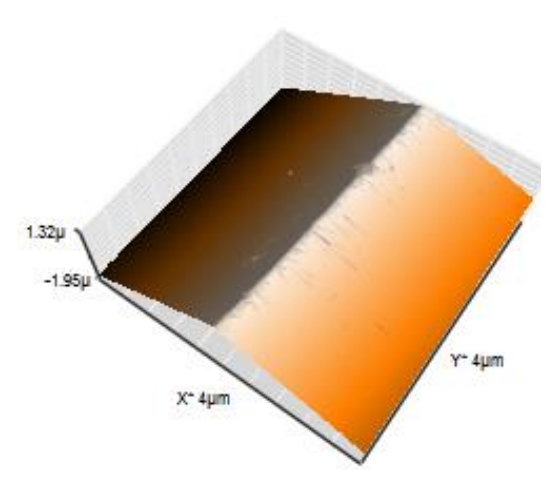

b)

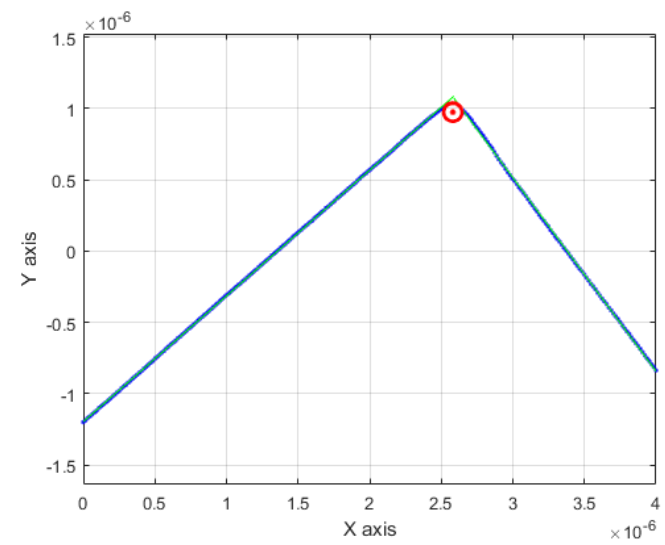

Fig. 7 Evaluation of cutting edge radius by AFM: a) 3D topography of tool cutting edge; b) 2D cross-section for reading cutting edge radius.

\subsection{Selection of cutting parameters}

Cutting experiments were carried out on a home-made ultra-precision machine tool. To validate the inhibitory effect of different cutting fluids on the wear of micro diamond tool, the 
spindle speed, cutting depth, and feed rate were invariable. The spindle speed was set to $1000 \mathrm{r} / \mathrm{min}$. The cutting depth and feed rate were configured as $2 \mu \mathrm{m}$ and $2 \mu \mathrm{m} / \mathrm{r}$. Four different cutting fluids were employed. Moreover, a 6061 aluminum alloy substrate with a diameter of $100 \mathrm{~mm}$ was employed as the workpiece.

When finishing the whole end face of the substrate, the cutting distance of tool is calculated as

$$
L=\frac{\pi\left(r_{1}^{2}\right)}{f \times 10^{3}}
$$

where $r_{l}$ is the diameter of the workpiece. $f$ is the feed rate. $L$ is the cutting distance.

\section{Results and discussions}

In diamond turning, the machined surface roughness is an important index to evaluate the performance of diamond tool. The criterion that a diamond tool can not be used is that the machined surface roughness exceeds the assigned value. In general, the wear of diamond tool has a significant influence on the achieved surface roughness [26].

To inhibit the wear of diamond tool, many solutions had been proposed, e.g. gas shielding, ultra-low temperature cooling, ultrasonic vibration-assisted cutting [27], surface modification, MQL, and etc. The MQL has the best effect in inhibiting the wear of diamond tool with the satisfactory cutting efficiency and workpiece accuracy. Therefore, in this work the MQL method was used to increase the life of micro diamond tool.

The cutting fluids can be classified into two types, cooling and lubricating. As well known, the wear mechanism of diamond tool heavily depends on the material properties of the workpiece, which leads to different inhibition effects of different cutting fluids on the wear of diamond tool. Using a suitable cutting fluid in response to the wear mechanism is of significant benefit to inhibit the wear of diamond tool.

The cutting distance related wear band width on the flank face is usually adopted to 
characterize the wear of diamond tool. The width of wear band can be deduced from the actual profile of the worn cutting edge acquired by the DTRC microscope system. Fig. 8 and Fig. 9 present the top view of the worn cutting edge and the polar diagram of the cutting edge waviness, respectively. It can be seen that the blue curve coupled with waviness in Fig. 9 has the similar variation tendency with the SEM observed contour of the worn cutting edge in Fig. 8. Therefore, it is possible to measure the radial wear length and the wear band length from the DTRC acquired cutting edge profile.

As shown in Fig. 9, the wear band length $l$ is first measured. The worn cutting edge (blue curve) intersects the ideal cutting edge at points A and B. As defined in Fig. 6(b), the ideal cutting edge is fitted with the least square circle method, yielding the mean tool nose radius, and the worn cutting edge fluctuates around the ideal cutting edge. The wear band length can be approximated as the length of arc $\mathrm{AB}$. The radial wear length $b$ is measured as the peakvalley value of the fluctuation of the worn cutting edge, i.e. the difference between the smallest tool nose radius $r_{1}$ and the largest tool nose radius $r_{2}$. By using the measured wear band length $l$ and radial wear length $b$, the wear band width can be predicted in terms of the geometries of tool tip. Finally, the wear volume can be calculated with the known width and length of the wear band. The measured results of tool cutting edge waviness at different cutting distances are shown in Fig. 10. 


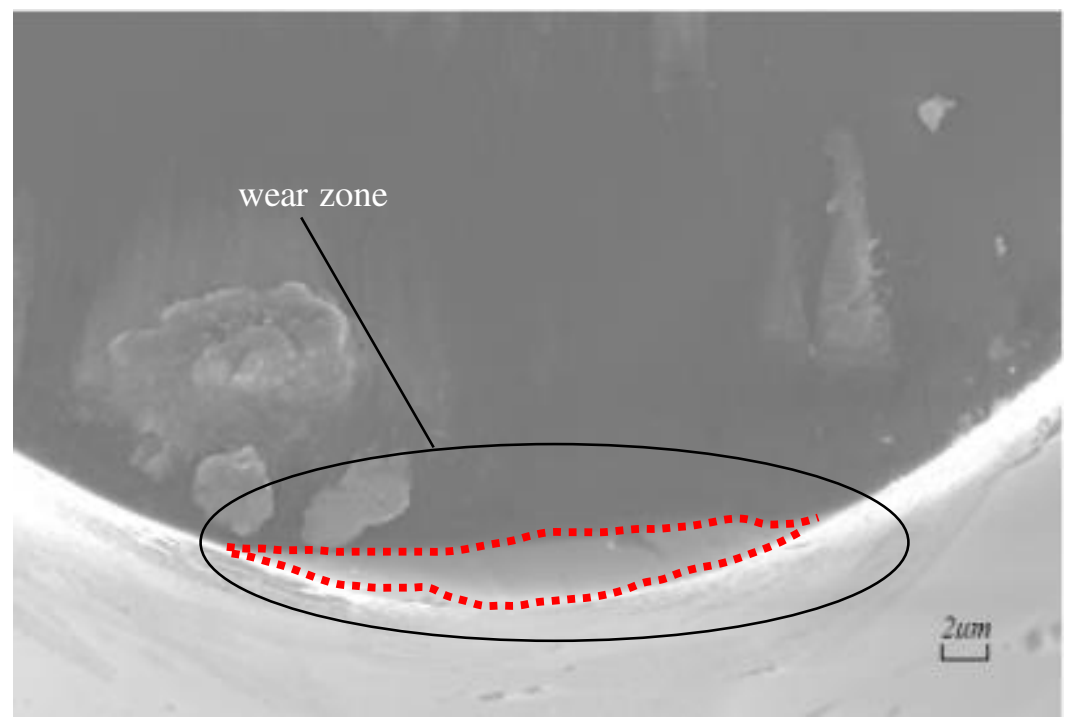

Fig. 8 The worn cutting edge profile of the test micro diamond tool observed with a scanning electron microscope.

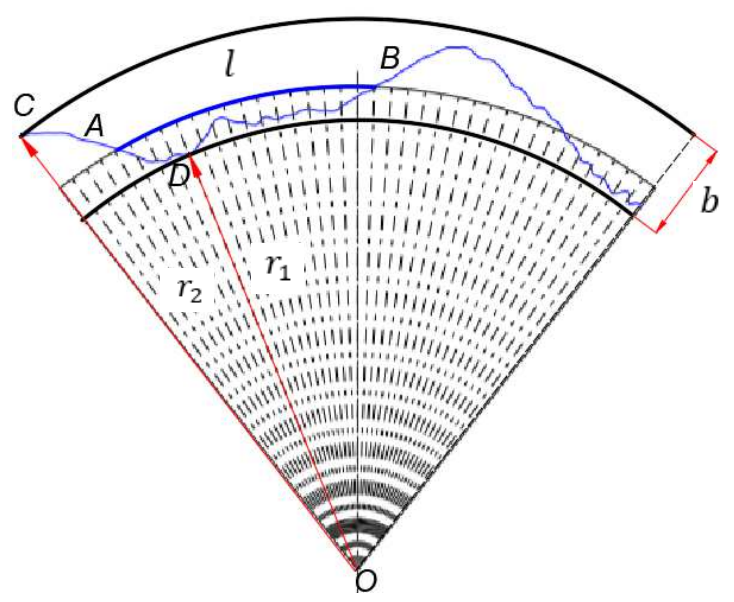

Fig. 10 Diagram of the method to measure the wear band length and the radial wear length. 


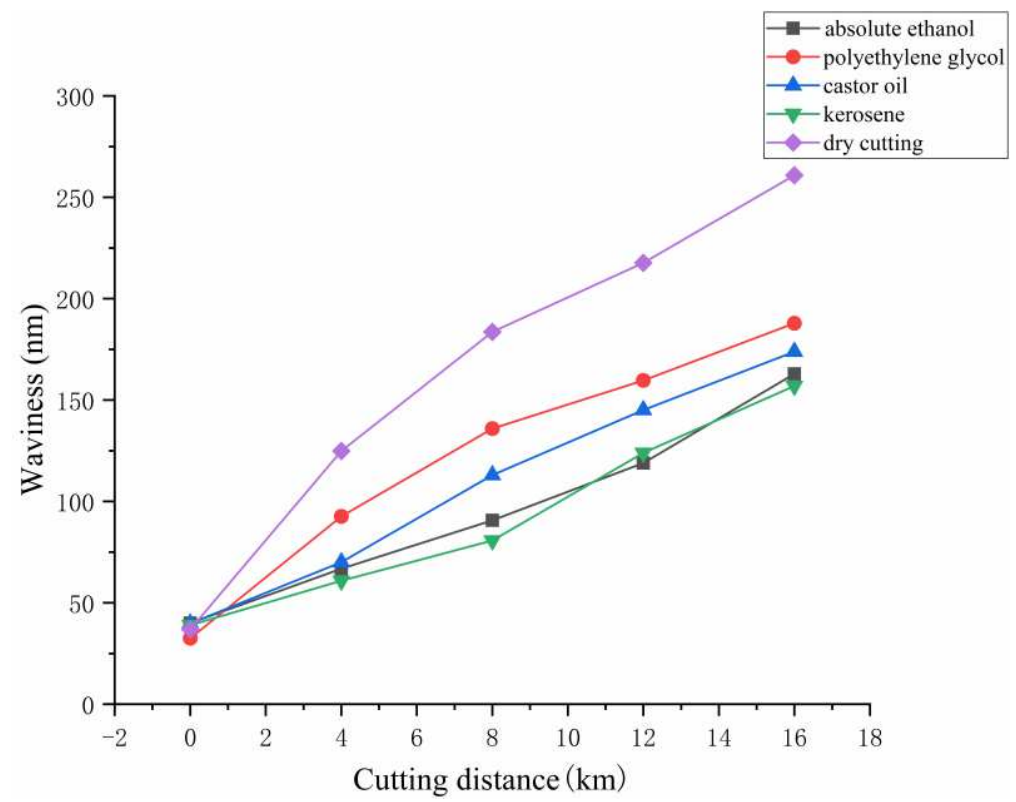

Fig. 10 The measured cutting edge waviness vs. cutting distance of micro diamond tool under different cutting fluids.

Based on the Usui wear rate model, i.e. Eq. (29), and the measured cutting edge waviness of the worn tool shown in Fig.10, the wear volumes of the worn tool at different cutting distances can be calculated, which are summarized in Fig. 11. It can be seen from Fig. 11 that the theoretical wear volume is always larger than the experimental wear volume. This is because the convective heat transfer of cutting fluid is not considered when modelling the temperature field on tool rake face in this work. As a result, the actual tool temperature is smaller than the predicted temperature, which in turn results in the actual wear volume being smaller than the theoretical wear volume. However, the predicted trend of tool wear volume under different cutting fluids is well consistent with the experimental one. 
a)

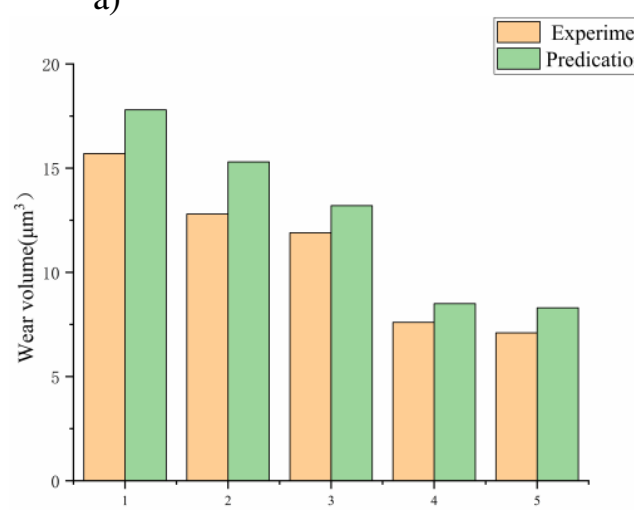

c)

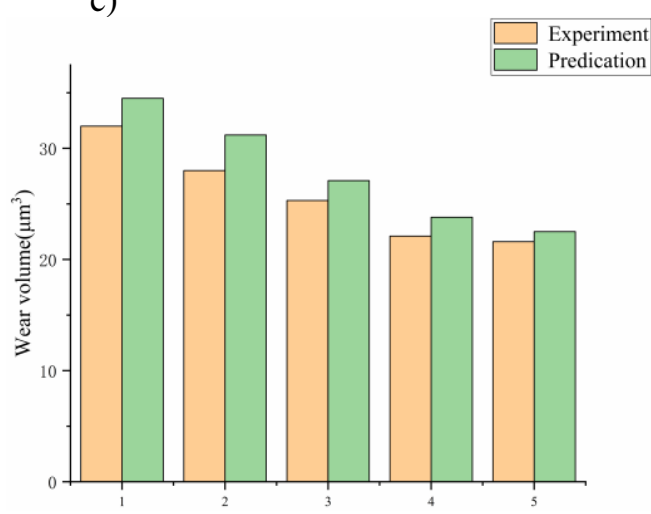

b)

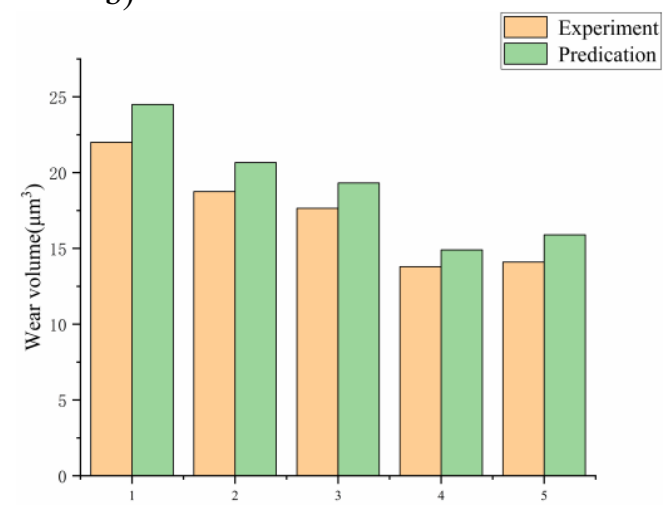

d)

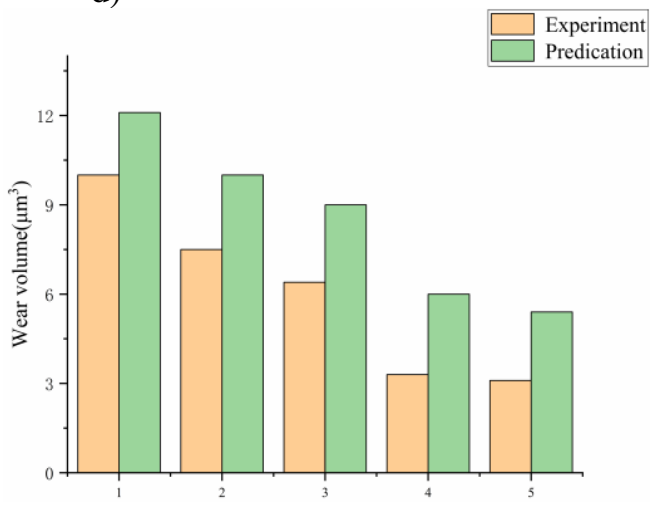

Fig. 11 Comparison of predicted and experimental wear volumes at different at different cutting distances :a) 4km; b) $8 \mathrm{~km}$; c) 12km; d) 16km. In the Fig. 12, 1 - Dry cutting; 2 - Polyethylene glycol; 3 - Castor oil; 4 - Absolute ethanol; 5 - Kerosene.

In order to reveal the underlying mechanism of tool wear inhibition by different cutting fluids, Raman spectroscopy and EDS analysis on the wear areas in response to dry cutting and MQL conditions were carried out. When tool cutting distance is $10 \mathrm{~km}$, the results of Raman spectrum analysis are shown in Fig. 12. 

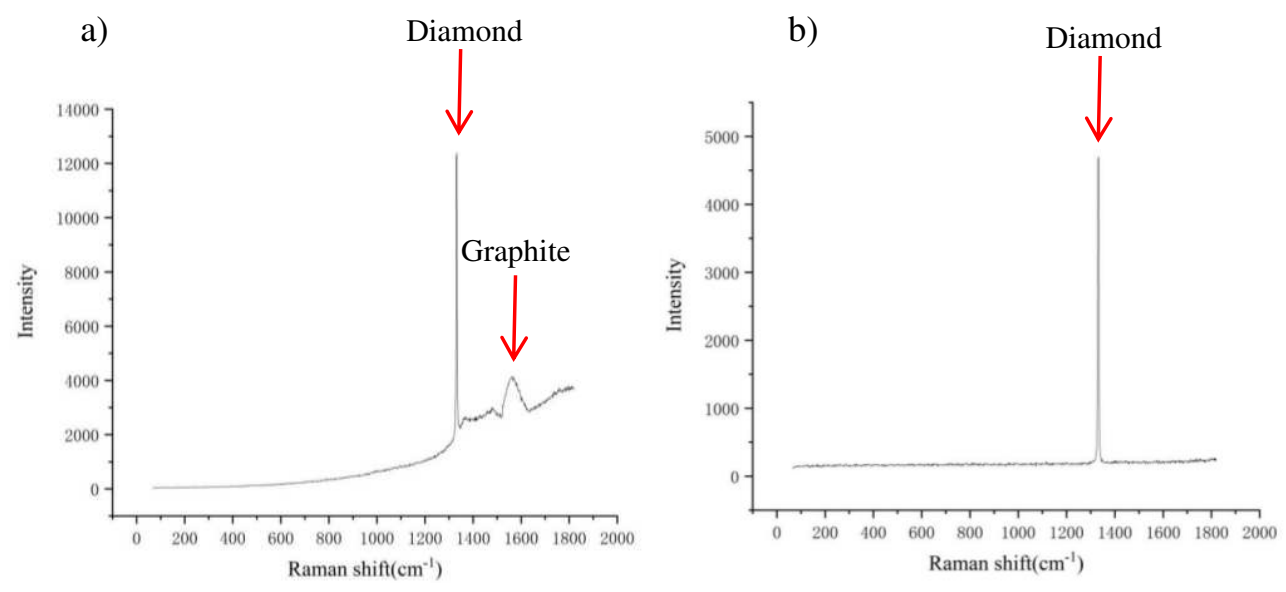

Fig.12. Raman spectrum analysis results of tool wear region under dry and MQL cutting conditions:

a) dry cutting; b) MQL cutting.

In Fig. 12, it can be seen that the graphite wear takes place on diamond tool surface in dry cutting, while no graphite wear occurs under MQL condition. The experimental observations validate that the appropriate use of cutting fluid can effectively slow down the graphitic wear of diamond tool.

Fig. 13 shows the EDS analysis results of the wear zone produced under dry cutting and MQL cutting. Table 2 presents the element sorts and contents of the wear zone. The EDS analysis results acquired at different points almost have no difference, i.e. that only the carbon (99.01\%) and oxygen $(0.99 \%)$ elements appear on the wear zone, regardless of the usage of cutting fluid. Such observations reveal that, in cutting of 6061 aluminum alloy with micro diamond tool, the diffuse wear does not occur on diamond tool surface in both dry cutting and MQL cutting. 
a)

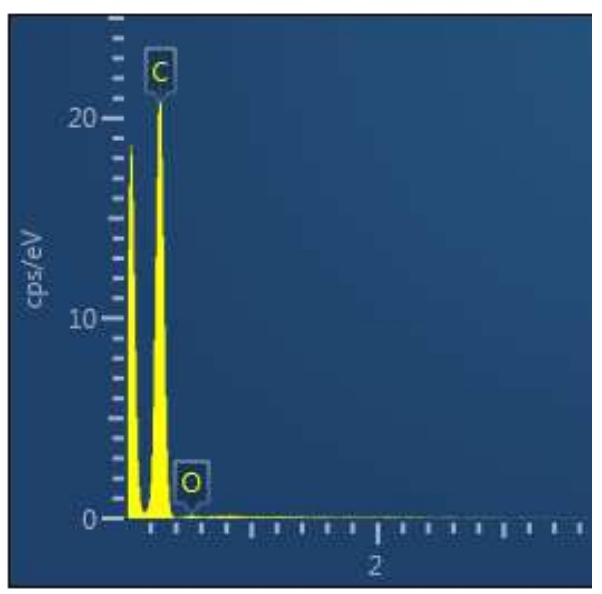

b)

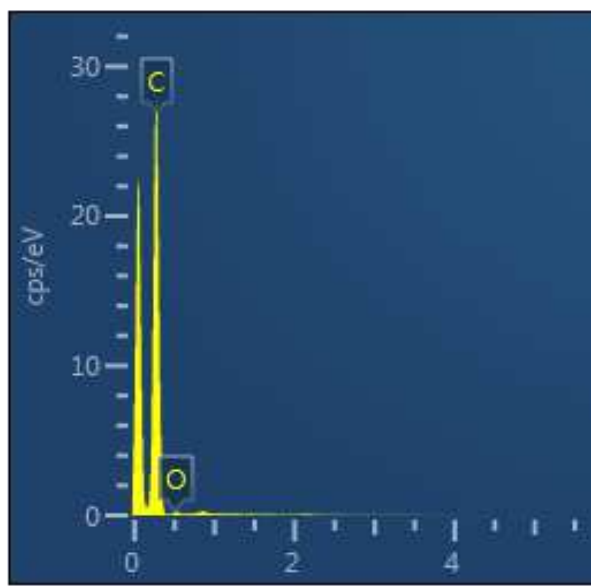

Fig.13 EDS analysis of the wear zone: a) MQL; b) dry cutting.

Table 2 Chemical element content of the wear region.

\begin{tabular}{ccccc}
\hline Chemical element & Line type & $\mathrm{Wt} \%$ & $\mathrm{Wt} \%$ Sigma & Atomic percentage \\
\hline $\mathrm{C}$ & K-Line & 98.69 & 0.26 & 99.01 \\
$\mathrm{O}$ & K-Line & 1.31 & 0.26 & 0.99 \\
Total Quantity: & & 100.00 & & 100.00 \\
\hline
\end{tabular}

All the experimental results demonstrate that in dry cutting of 6061 aluminum alloy, the diamond tool mainly suffers from the graphitization wear and scratch wear. The factors responsible for the graphitization of diamond include the high stress and temperature. Under MQL condition, the cutting fluid can relief the friction at the tool-chip contact surface, which in turn reduces the friction force applied to tool surface. As a result, the stress distributed on the cutting region can be effectively reduced. On the other hand, the cutting fluid can take away some of the heat by means of thermal convection, which reduce the temperature on the rake face. Consequently, no graphite wear is observed on the micro diamond tool in MQL cutting.

\section{Conclusion}

This work aims to establish the temperature field model of micro diamond tool under the condition of minimum quantity lubrication, and optimize the cutting fluid in inhibiting the wear of micro diamond tool. According to the theoretical modelling, related analyses and experimental validations, some important conclusions can be drawn as follows. 
(1) Based on the Fourier heat conduction theory, a cutting temperature field model is firstly established to solve the maximum temperature on tool rake face under dry cutting condition. Secondly, regarding the boundary film as a layer of heat-conduction medium, the cutting temperature field model is further modified to predict the temperature on tool rake face under minimum quantity lubrication condition. The prediction results indicate that the minimum quantity lubrication method can effectively reduce the temperature on tool rake face.

(2) The maximum temperature on tool rake face under different cutting fluids is calculated with the well-established cutting temperature field model. The results demonstrate that the lower the viscosity of the cutting fluid, the more effective the cutting fluid in reducing tool temperature. Low viscosity lubricant fluids have high wettability (small contact angle), higher mist particle concentration and larger mist particle diameter. Despite their relatively low thermal conductivity, the larger mist particle diameter and higher mist particle concentration reduce surface roughness and inhibit tool wear

(3) The wear volumes of micro diamond tool are calculated in terms of the predicted cutting temperature and Usui wear rate model, and the variation trend of which agree well with the experimental results. Such satisfactory consistency confirms that the cutting fluid can effectively reduce the cutting temperature and inhibit the wear of tool. Raman spectrum analysis further reveals that the graphite wear of micro diamond tool inevitably takes place in dry cutting, and the contribution of cutting fluid is to slow down the graphitization of diamond. The results of EDS analysis indicate that no diffusion wear occur on the micro diamond tool in dry or minimum quantity lubrication cutting of 6061 aluminum alloy.

\section{Acknowledgment}

The authors would like to thank the Science Challenge Project (No. TZ2018006) for the support of this work. 


\section{Availability of data and materials}

All data and materials used to produce the results in this article can be obtained upon request from the corresponding authors.

\section{Declarations}

Ethical approval The authors declare that there is no ethical issue applied to this article.

Consent to participate The authors declare that all authors have read and approved to submit this manuscript to IJAMT.

Consent to publish The authors declare that all authors agree to sign the transfer of copyright for the publisher to publish this article upon on acceptance.

Competing interests The authors declare that there is no conflict of interest.

\section{References}

[1] S. J. Zhang, Z. W. Xu, F. Z. Fang, Fabrication of Micro Tool Using Focused Ion Beam Milling Technique, Journal of Tianjin University, 43 (2010) 469-472.

[2] S. Zhang, Z. Liu, A new approach to cutting temperature prediction considering the diffusion layer in coated tools, International Journal of Machine Tools and Manufacture, 49 (2009) 619-624.

[3] Z. C. Lin, M. H. Lin, Y. C. Hsu, Simulation of temperature field during nanoscale orthogonal cutting of single-crystal silicon by molecular statics method, Computational Materials Science, 81 (2014) 58-67.

[4] Z. Zhang, D. Zhao, Y. Zhao, Simulation and experimental study on temperature and stress field of fullsized PDC bits in rock breaking process, Journal of Petroleum Science and Engineering, 186 (2019) 1-12.

[5] R. Komanduri, Z. B. Hou, Thermal modeling of the metal cutting process: Part I- Temperature rise distribution due to shear plane heat source, International Journal of Mechanical Sciences, 42 (2000) 17151752.

[6] N. A. Abukhshim, P. T. Mativenga, M A Sheikh, Investigation of heat partition in high speed turning of high strength alloy steel, International Journal of Machine Tools and Manufacture, 45 (2005) 1687-1695.

[7] W. Grzesik, M. Bartoszuk, P. Nieslony, Finite difference analysis of the thermal behaviour of coated tools in orthogonal cutting of steels, International Journal of Machine Tools and Manufacture, 44 (2004) 1451- 
1462.

[8] S. Wang, S. To, C. Y. Chan, A study of the cutting-induced heating effect on the machined surface in ultraprecision raster milling of $6061 \mathrm{Al}$ alloy, The International Journal of Advanced Manufacturing Technology, $51(2010) 69-78$.

[9] W. Sawangsri, K. Cheng, An experiment-based investigation on the partitioned distri- bution of cutting heat and cutting temperature in micro cutting, 13 (2014) 173-195.

[10] M. A. Davies, T. Ueda, M. Saoubi R, On The Measurement of Temperature in Material Removal Processes, CIRP Annals Manufacturing Technology, 56 (2007) 581-604.

[11] J. F. Zhao, Z. Q. Liu, PVD AlTiN coating effects on tool-chip heat partition coefficient and cutting temperature rise in orthogonal cutting Inconel 718, International Journal of Heat and Mass Transfer, 163 (2020) 1-12.

[12] H. Puls, F. Klocke, D. Veselovac, FEM-based prediction of heat partition in dry metal cutting of AISI 1045, International Journal of Advanced Manufacturing Technology, 86 (2015) 1-9.

[13] S. H. You, J. H. Lee, A Study on Cutting Characteristics in Turning Operations of Titanium Alloy used in Automobile, International Journal of Precision Engineering and Manufacturing, 20 (2019) 209-216.

[14] G. Hao, Z. Liu, X. Liang, Influences of TiAlN Coating on Cutting Temperature during Orthogonal Machining H13 Hardened Steel, Coatings, 9 (2019) 1-14.

[15] G. Su, X. Xiao, J. Du, On cutting temperatures in high and ultrahigh-speed machining, International Journal of Advanced Manufacturing Technology, 107 (2020) 73-83.

[16] X. X. Meng, Y. X. Lin, Chip morphology and cutting temperature of ADC12 aluminum alloy during high-speed milling, Rare Metals, 3 (2020) 1915-1923.

[17] U. Karaguzel, E. Budak, Investigating effects of milling conditions on cutting temperatures through analytical and experimental methods, Journal of Materials Processing Technology, 262 (2018) 532-540.

[18] W. Grzesik, Composite layer-based analytical models for tool-chip interface temperatures in machining medium carbon steels with multi-layer coated cutting tools, Journal of Materials Processing Technology, 176 (2006) $102-110$.

[19] N. Shreyes, Melkote, W. Grzesik, Advances in material and friction data for modelling of metal machining, CIRP Annals Manufacturing Technology 66 (2017) 731-754.

[20] H. Xiao, S. Liu, 2D nanomaterials as lubricant additive: A review, Materials and Design, 135 (2017) 319-332. 
[21] J. Wang, J. K. Carson, M. F, A new approach to modelling the effective thermal conductivity of heterogeneous materials, International Journal of Heat and Mass Transfer, 49 (2006) 3075-3083.

[22] X. Li, The effect of coolant flow rate on cooling in machining, Technical Papers of the NAMRI, 120 (1995) 109-114.

[23] C. L. He, W. J. Zong, T Sun, Origins for the size effect of surface roughness in diamond turning, International Journal of Machine Tools and Manufacture, 106 (2016) 22-42.

[24] J. Guo, J. Zhang, R. Kang, A critical review on the chemical wear and wear suppression of diamond tools in diamond cutting of ferrous metals, International Journal of Extreme Manufacturing, 2 (2019) 1-41. [25] T. Kitagawa, K. Maekawa, E. Usui, Analytical Prediction of Flank Wear of Carbide Tools in Turning Plain Carbon Steels (Part 1)-Characteristic Equation of Flank Wear, Bulletin of the Japan Society of Precision Engineering, 22 (1988) 263-269. 